\title{
ANÁLISE DA SITUAÇÃO EM SAÚDE: A MORTALIDADE FETAL NA 10ª REGIÃO DE SAÚDE DO CEARÁ
}

\section{Health situation analysis: fetal mortality in the 10th health region of Ceará \\ Análisis de la situación de salud: la mortalidad fetal de la 10 ${ }^{a}$ región de salud de Ceará}

\author{
Katherine Jeronimo Lima \\ Universidade Estadual do Ceará - UECE - Fortaleza (CE) - Brasil \\ Cristianne Soares Chaves \\ $10^{\mathrm{a}}$ Coordenadoria Regional de Saúde do Ceará - CRES - Limoeiro do Norte (CE) - Brasil
}

Edsângelo de Oliveira Gomes

$10^{\mathrm{a}}$ Coordenadoria Regional de Saúde do Ceará - CRES - Limoeiro do Norte (CE) - Brasil

Maria Aparecida de Lima

Escola de Saúde Pública do Ceará - ESP - Fortaleza (CE) - Brasil

Elanny Cristina Pascôa Candeira

Escola de Saúde Pública do Ceará - ESP - Fortaleza (CE) - Brasil

\section{Fiama Kécia Silveira Teófilo}

Universidade Estadual do Ceará - UECE - Fortaleza (CE) - Brasil

\section{Glaucilândia Pereira Nunes}

Universidade Estadual do Ceará - UECE - Fortaleza (CE) - Brasil

Radmila Alves Alencar Viana

Universidade Estadual do Ceará - UECE - Fortaleza (CE) - Brasil

\section{RESUMO}

Objetivo: Analisar a situação de saúde, no contexto da mortalidade fetal, na $10^{\mathrm{a}}$ Região de Saúde do Ceará. Métodos: Estudo transversal, descritivo, retrospectivo, com informações referentes a 71 óbitos fetais de mães residentes da $10^{\mathrm{a}}$ Região de Saúde do Ceará, ocorridos no período de 2012 a 2013. Utilizaram-se dados do Sistema de Informação sobre Mortalidade e das Fichas de Investigação do Óbito Fetal, que foram analisados em frequências absolutas e relativas. Resultados: Observou-se que 52,1\% $(n=37)$ das mortes fetais ocorreram em mulheres com menos de 30 anos. Das mães, 35,2\% $(n=25)$ eram primigestas, 49,3\% $(n=35)$ iniciaram o pré-natal antes de 12 semanas gestacionais e 64,7\% (n=46) tiveram complicação na gestação. Dos partos, 36,6\% $(n=26)$ deles ocorreram entre 37 e 41 semanas de gestação, e 67,6\% $(n=48)$ por via vaginal. Dos natimortos, $31 \%(n=22)$ tinham peso acima de $2.500 \mathrm{~g}$, e $38 \%(n=28)$ tiveram hipóxia intrauterina como causa básica da morte. Conclusão: A situação de saúde quanto à mortalidade fetal evidenciou alta taxa de óbitos no ano de 2013 (14,5/1.000 nascimentos), cujas mães eram jovens, primigestas e com complicações gestacionais, com mortes ocorridas antes do parto, baixo peso e hipóxia intrauterina.

Descritores: Morte fetal; Assistência Pré-Natal; Assistência à Saúde.

\section{ABSTRACT}

Objective: To analyze the health situation, in the context of fetal mortality, in the 10th Health Region of Ceará. Methods: Cross-sectional, descriptive and retrospective study with information regarding 71 fetal deaths of mothers living in Limoeiro do Norte 10th Health Region of Ceará, which occurred in the period from 2012 to 2013. The study used data from the Brazilian Mortality Information System (SIM) and the Fetal Death Investigation Reports, which were analyzed in absolute and relative frequencies. Results: It was observed that 52.1\% $(n=37)$ of fetal deaths occurred in women aged under 30 years. Of the mothers, $35.2 \%(n=25)$ were in their first pregnancy; 49.3\% $(n=35)$ started prenatal care before 12 gestational weeks, and $64.7 \%(n=46)$ experienced complications during pregnancy. Of the deliveries, 36.6\% $(n=26)$ occurred between 37 and 41 weeks of gestation, and $67.6 \%(n=48)$ were vaginal delivery. Of the stillborns, 31\% $(n=22)$ had weight above 2,500g, and 38\% (n=28) had intrauterine hypoxia as the underlying cause of death. Conclusion: The health situation regarding fetal mortality showed a high death rate in the year 2013 (14.5/1,000 births), whose mothers were young, in their first pregnancy and experiencing gestational complications, with deaths occurring before delivery, low birth weight and intrauterine hypoxia.

Descriptors: Fetal Death; Prenatal Care; Delivery of Health Care. 


\section{RESUMEN}

Objetivo: Analizar la situación de salud en el contexto de la mortalidad fetal de la $10^{a}$ Región de Salud de Ceará. Métodos: Estudio transversal, descriptivo y retrospectivo con informaciones de 71 óbitos fetales de madres residentes de la $10^{a}$ Región de Salud de Ceará en el periodo entre 2012 y 2013. Se utilizaron los datos del Sistema de Información sobre Mortalidad y de las Fichas de Investigación del Óbito Fetal que fueron analizados en frecuencias absolutas y relativas. Resultados: Se observó que el 52,1\% (n=37) de las muertes fetales se dieron en mujeres con menos de 30 años. Entre las madres, el 35,2\% $(n=25)$ eran primigestas, el 49,3\% (n=35) iniciaron el prenatal antes de las 12 semanas de gestación y el 64,7\% (n=46) tuvieron complicaciones en la gestación. Entre los partos, el 36,6\% $(n=26)$ se dieron entre 37 y 41 semanas de gestación y el 67,6\% $(n=48)$ por vía vaginal. Entre los natimuertos, el $31 \%(n=22)$ tenían más de $2.500 \mathrm{~g}$ de peso y el $38 \%(n=28)$ tuvieron hipoxia intrauterina como causa básica de muerte. Conclusión: La situación de salud respecto la mortalidad fetal evidenció elevada tasa de óbitos en el año de 2013 (14,5/1.000 nacimientos) cuyas madres eran jóvenes, primigestas, y con complicaciones en la gestación, con muertes antes del parto, bajo peso e hipoxia intrauterina.

Descriptores: Muerte Fetal; Atención Prenatal; Prestación de Atención de Salud.

\section{INTRODUÇÃO}

As denominações óbito fetal, morte fetal, nascido morto/natimorto são definidas como a morte de um produto da concepção, antes da expulsão ou da extração completa do corpo da mãe, independentemente da duração da gravidez. A morte do feto caracteriza-se pela inexistência, depois da separação, de qualquer sinal descrito para o nascido vivo ${ }^{(1)}$. No Brasil, determina-se como óbito fetal o feto com peso ao nascer igual ou inferior a 500 gramas, idade gestacional de 22 semanas (154 dias) e/ou conceptos com comprimento corpóreo de 25 centímetros cabeça-calcanhar ou mais ${ }^{(2)}$.

Estima-se que, por ano, aconteçam 2,6 milhões de mortes fetais no mundo, sendo a quase totalidade delas (98\%) ocorridas em países em desenvolvimento( ${ }^{(3)}$.

No Brasil, os óbitos fetais ocorrem com frequência elevada, chegando a superar em números os óbitos neonatais (ocorridos nos primeiros 28 dias de vida) $)^{(4)}$. A região Nordeste, no ano de 2012, apresentou taxa da mortalidade fetal de 13,4 por 1.000 nascimentos, sendo a maior do país. O estado do Ceará, no mesmo período, apresentou valor de 12,6 por mil nascimentos, enquanto a média nacional esteve em 11/1.000 nascimentos ${ }^{(5,6)}$.

$\mathrm{Na} 10^{\mathrm{a}}$ Região de Saúde do Ceará, localizada em Limoeiro do Norte, observou-se o crescente número de óbitos fetais ao longo dos anos. Uma análise realizada na referida região mostrou que houve uma redução de $20,8 \%$ do número dos óbitos infantis nos anos de 2009 a 2013. No entanto, houve um crescimento de $15 \%$ nos óbitos fetais no decorrer deste período ${ }^{(6)}$.

Ao longo das últimas décadas, a redução dos óbitos infantis configurou-se como uma das prioridades entre as políticas públicas em diversos países ${ }^{(7)}$. Porém, o mesmo não pode ser afirmado para o óbito fetal, que ainda não dispõe de um importante reconhecimento na agenda de saúde do mundo e tampouco foi preocupação entre os Objetivos do Milênio da Organização das Nações Unidas $(\mathrm{ONU})^{(8,9)}$.

A natimortalidade é considerada indicador de saúde sensível para avaliar as condições de vida de uma população, uma vez que reflete o estado de saúde da mulher, a qualidade e a acessibilidade dos cuidados primários de saúde disponibilizados à gestante, bem como, a qualidade da assistência intraparto ${ }^{(10,11)}$.

A etiologia do óbito fetal é multifatorial, envolvendo fatores inter-relacionados que incluem condição socioeconômica, fatores biológicos e pertinentes à qualidade da assistência à saúde ${ }^{(11-13)}$.

A mortalidade fetal é um problema da saúde materno-infantil pouco estudado, apesar das mortes fetais serem influenciadas pelas mesmas circunstâncias e etiologias que a mortalidade neonatal precoce. Além disso, tem sido dada pouca relevância às causas desses óbitos, visto que, se associa a sua ocorrência como uma eventualidade fatal ${ }^{(1,9,10,14)}$.

O natimorto é um dos resultados adversos mais importantes, no entanto, ainda mal compreendido e reconhecido na gravidez ${ }^{(9)}$. Pelo fato de não se tratar de um indicador de saúde, os óbitos fetais têm sido historicamente negligenciados pelos serviços de saúde, não sendo incorporados nas rotinas de trabalho, nas discussões e nas análises sobre sua ocorrência, tampouco são destinados investimentos específicos para redução da natimortalidade ${ }^{(1,10)}$.

Diante do contexto apresentado, o presente estudo objetivou analisar a situação da saúde, no contexto da mortalidade fetal, na $10^{\mathrm{a}}$ Região de Saúde do Ceará. 


\section{MÉTODOS}

Estudo transversal, descritivo, retrospectivo, realizado na $10^{a}$ Região de Saúde do Ceará, que pertence a uma das 22 regiões de saúde do modelo de atenção à saúde do estado do Ceará, sendo composta por 11 municípios, conforme o Plano Diretor de Regionalização (PDR) de 2014, e possui população total de 220.167 habitantes $^{(15,16)}$.

As Regiões de Saúde são espaços geográficos contínuos, constituídos por agrupamento de municípios limítrofes, delimitadas a partir de identidades culturais, econômicas e sociais, e de redes de comunicação e infraestrutura de transportes compartilhados, com a finalidade de integrar a organização, o planejamento e a execução de ações e serviços de saúde(17).

Selecionaram-se para população do estudo todos os óbitos fetais de mães residentes dos onze municípios abrangentes da referida Região de Saúde no período de 01/01/2012 a 31/12/2013.

Utilizou-se como fonte de dados a Declaração de Óbito (DO), proveniente do Sistema de Informação sobre Mortalidade (SIM). No entanto, existem informações que não constam na DO, como antecedentes da mãe, acompanhamento no pré-natal e assistência ao parto, consideradas importantes para realização do presente estudo. Desse modo, acrescentou-se também o uso das Fichas de Investigação do Óbito Infantil e Fetal, um instrumento do Ministério da Saúde para fortalecimento da vigilância do óbito ${ }^{(1)}$.

A escolha dessas fichas ocorreu devido a fornecerem informações sobre a assistência ao pré-natal, à gestação, ao parto e ao nascimento, as quais são compostas de: Fichas de Investigação do Óbito Fetal - Serviço de saúde: ambulatorial e hospitalar e Ficha de Investigação do Óbito Infantil - Entrevista domiciliar, as quais são sumarizadas na Ficha de Investigação do Óbito Infantil e Fetal - Síntese, Conclusões e Recomendações ${ }^{(1)}$.

A coleta de dados ocorreu nos meses de março a abril de 2015, por meio de visitas ao setor de Vigilância Epidemiológica da Secretaria Municipal de Saúde dos onze municípios pertencentes à referida Região de Saúde. Para obtenção dos dados, utilizou-se um formulário semiestruturado contendo as variáveis sociodemográficas da mãe (idade e escolaridade), variáveis gestacionais (história gestacional anterior, início do pré-natal, complicação na gestação e tipo de complicação), variáveis do parto (motivo pelo qual buscou atendimento na hora do parto, duração da gestação em semanas e tipo do parto), e variáveis do natimorto (morte em relação ao parto, peso ao nascer e causa básica da morte).

Os dados obtidos através da DO e das Fichas de Investigação de Óbito Fetal foram transportados para um banco de dados e analisados em frequências absolutas e relativas com uso do programa Epi-Info, versão 7.1.2.0.

Este estudo recebeu aprovação do Comitê de Ética em Pesquisa da Escola de Saúde Pública do Ceará, sob parecer n. 958.482, de acordo com a Resolução n. ${ }^{\circ} 466 / 12$.

\section{RESULTADOS}

No período estudado, identificou-se a ocorrência de 71 óbitos fetais na $10^{\mathrm{a}}$ Região de Saúde do Ceará. Dos onze municípios que abrangem a região, somente um município não apresentou ocorrência de óbito fetal nos anos pesquisados.

Em 2012, os óbitos fetais corresponderam a 43,7\% $(n=31)$ do total analisado, enquanto no ano de 2013 , o percentual foi de 56,3\% $(\mathrm{n}=40)$. Ao calcular a taxa de mortalidade fetal, encontrou-se, em 2012, uma taxa de 11 óbitos fetais por 1.000 nascimentos, e de 14,5 óbitos por 1.000 nascimentos no ano de 2013.

Sobre as informações sociodemográficas inerentes às mães, observou-se que $52,1 \%(n=37)$ das mortes fetais ocorreram em mulheres com a faixa etária de 20 a 30 anos. Destacam-se também as mães com a idade de 35 anos ou mais, as quais corresponderam a 18,3\% $(n=13)$. Sobre a escolaridade, mais da metade dos natimortos, 53,5\% $(n=38)$, ocorreu em mulheres com 8 a 11 anos de estudo (Tabela I).

Tabela I - Distribuição e frequência dos óbitos fetais segundo variáveis sociodemográficas da mãe. $10^{\mathrm{a}}$ Região de Saúde do Ceará, Brasil, 2012 a 2013.

\begin{tabular}{|c|c|c|c|c|c|c|}
\hline \multirow{2}{*}{ Variáveis } & \multicolumn{2}{|c|}{2012} & \multicolumn{2}{|c|}{2013} & \multicolumn{2}{|c|}{ Total } \\
\hline & $\mathbf{n}$ & $\%$ & $\mathbf{n}$ & $\%$ & $\mathbf{n}$ & $\%$ \\
\hline \multicolumn{7}{|c|}{ Idade da mãe (em anos) } \\
\hline$<19$ & 5 & 16,1 & 5 & 12,5 & 10 & 14,0 \\
\hline $20-30$ & 14 & 45,1 & 23 & 57,5 & 37 & 52,1 \\
\hline $31-34$ & 3 & 9,7 & 4 & 10,0 & 7 & 9,9 \\
\hline $35 \mathrm{e}+$ & 7 & 22,6 & 6 & 15,0 & 13 & 18,3 \\
\hline Sem informação & 2 & 6,5 & 2 & 5,00 & 4 & 5,7 \\
\hline \multicolumn{7}{|c|}{ Escolaridade (em anos de estudo) } \\
\hline Nenhuma & 1 & 3,2 & 1 & 2,50 & 2 & 2,82 \\
\hline $1-3$ & 3 & 9,7 & 1 & 2,50 & 4 & 5,63 \\
\hline $4-7$ & 8 & 25,8 & 12 & 30,00 & 20 & 28,2 \\
\hline $8-11$ & 16 & 51,6 & 22 & 55,00 & 38 & 53,5 \\
\hline Sem informação & 3 & 9,7 & 4 & 10,00 & 7 & 9,9 \\
\hline
\end{tabular}

Fonte: Sistema de Informação sobre Mortalidade. Fichas de Investigação do Óbito Fetal. 
As variáveis relacionadas à gestação apontaram maior constância de mortes fetais em primigestas (primeira gestação), com $35,2 \%(n=25)$. A frequência de mulheres com história gestacional de natimorto constituiu-se em $28,2 \%$ ( $n=20)$. Do total de mães, 49,3\% $(\mathrm{n}=35)$ realizou a primeira consulta de pré-natal antes de 12 semanas de gestação. Sobre as complicações presentes no período gestacional, verificou-se que $64,7 \%(n=46)$ das mães tinham sido acometidas por alguma complicação. Além disso, observou-se que, do total de mulheres que apresentou complicações na gestação, 50,0\% (n=23) delas tiveram hipertensão gestacional (Tabela II).

Tabela II - Distribuição e frequência dos óbitos fetais segundo variáveis gestacionais. 10ª Região de Saúde do Ceará, Brasil, 2012 a 2013.

\begin{tabular}{|c|c|c|c|c|c|c|}
\hline \multirow[t]{2}{*}{ Variáveis } & \multicolumn{2}{|c|}{2012} & \multicolumn{2}{|c|}{2013} & \multicolumn{2}{|c|}{ Total } \\
\hline & $\mathbf{n}$ & $\%$ & $\mathbf{n}$ & $\%$ & $\mathbf{n}$ & $\%$ \\
\hline \multicolumn{7}{|c|}{ História Gestacional Anterior } \\
\hline Nascido vivo & 9 & 29,0 & 14 & 35,0 & 23 & 32,4 \\
\hline Natimorto & 8 & 25,8 & 12 & 30,0 & 20 & 28,2 \\
\hline Nenhuma & 12 & 38,7 & 13 & 32,5 & 25 & 35,2 \\
\hline Sem informação & 2 & 6,5 & 1 & 2,5 & 3 & 4,3 \\
\hline \multicolumn{7}{|c|}{ Iniciou o Pré-Natal (em semanas) } \\
\hline$\leq 12$ & 11 & 35,5 & 24 & 60,0 & 35 & 49,3 \\
\hline$\geq 13$ & 11 & 35,5 & 13 & 32,5 & 24 & 33,8 \\
\hline$\overline{\text { Sem informação }}$ & 9 & 29,0 & 3 & 7,5 & 12 & 16,9 \\
\hline \multicolumn{7}{|c|}{ Complicação na Gestação } \\
\hline Sim & 21 & 67,7 & 25 & 62,5 & 46 & 64,7 \\
\hline Não & 4 & 13,0 & 8 & 20,0 & 12 & 17,0 \\
\hline Sem informação & 6 & 19,3 & 7 & 17,5 & 13 & 18,3 \\
\hline \multicolumn{7}{|c|}{ *Tipo de Complicação } \\
\hline Hipertensão & 11 & 52,4 & 12 & 48,0 & 23 & 50,0 \\
\hline Infecção Urinária & 7 & 33,3 & 10 & 40,0 & 17 & 37,0 \\
\hline Diabetes & 1 & 4,8 & 2 & 8,0 & 3 & 6,5 \\
\hline Fator Rh negativo & 2 & 9,5 & 1 & 4,0 & 3 & 6,5 \\
\hline
\end{tabular}

Fonte: Sistema de Informação sobre Mortalidade. Fichas de Investigação de Óbito Fetal.

*Percentual calculado considerando somente o total de complicações informadas.

Com relação aos motivos da gestante para buscar atendimento na hora do parto, somente $69 \%$ (n=49) dos casos apresentaram informação para esta variável, sendo que $33,8 \%(n=24)$ das gestantes procuraram atendimento devido à ausência de movimentos fetais. Além disso, observou-se maior frequência de mortes fetais entre 37- 41 semanas de gestação, com percentual de 36,6\% $(n=26)$. Vale ressaltar que $41 \%(n=29)$ das mortes fetais ocorreram entre 28 e 36 semanas de gestação. Em referência ao tipo de parto, notou-se que $67,6 \%(n=48)$ dos partos ocorreram por via vaginal (Tabela III).

Tabela III - Distribuição e frequência dos óbitos fetais segundo variáveis do parto. $10^{\mathrm{a}}$ Região de Saúde do Ceará, Brasil, 2012 a 2013 .

\begin{tabular}{|c|c|c|c|c|c|c|}
\hline \multirow{2}{*}{ Variáveis } & \multicolumn{2}{|c|}{2012} & \multicolumn{2}{|c|}{2013} & \multicolumn{2}{|c|}{ Total } \\
\hline & $\mathbf{n}$ & $\%$ & $\mathbf{n}$ & $\%$ & $\mathbf{n}$ & $\%$ \\
\hline \multicolumn{7}{|c|}{ Motivo que buscou atendimento na hora do parto } \\
\hline Parou de mexer & 10 & 32,2 & 14 & 35,0 & 24 & 33,8 \\
\hline Contrações & 8 & 25,8 & 9 & 22,5 & 17 & 24,0 \\
\hline Sangramento & 4 & 13,0 & 6 & 15,0 & 10 & 14,1 \\
\hline Sem informação & 9 & 29,0 & 11 & 27,5 & 20 & 28,1 \\
\hline \multicolumn{7}{|c|}{ Duração da gestação (em semanas) } \\
\hline$<22$ & 1 & 3,2 & 0 & 0,0 & 1 & 1,4 \\
\hline $22-27$ & 3 & 9,7 & 12 & 30,0 & 15 & 21,0 \\
\hline $28-31$ & 5 & 16,1 & 7 & 17,5 & 12 & 17,0 \\
\hline $32-36$ & 7 & 22,6 & 10 & 25,0 & 17 & 24,0 \\
\hline $37-41$ & 15 & 48,4 & 11 & 27,5 & 26 & 36,6 \\
\hline \multicolumn{7}{|l|}{ Tipo de parto } \\
\hline Vaginal & 18 & 58,1 & 30 & 75,0 & 48 & 67,6 \\
\hline Cesárea & 11 & 35,5 & 9 & 22,5 & 20 & 28,2 \\
\hline Sem informação & 2 & 6,4 & 1 & 2,5 & 3 & 4,2 \\
\hline
\end{tabular}

Fonte: Sistema de Informação sobre Mortalidade. Fichas de Investigação de Óbito Fetal. 
Sobre as variáveis referentes ao natimorto, ao se analisar a morte fetal em relação ao parto, verificou-se que $83,1 \%$ ( $\mathrm{n}=59$ ) das mortes aconteceram antes do parto e 9,8\% $(\mathrm{n}=7)$ durante o trabalho de parto. Quanto ao peso ao nascer, constatou-se uma maior ocorrência dos óbitos fetais na faixa de peso superior a 2.500 gramas, com frequência de $31 \%$ ( $\mathrm{n}=22$ ). Contudo, a maioria dos natimortos $(63,3 \%)$ possuía baixo peso, sendo que 33,8\% (n=24) tinha peso acima de 1.500 gramas. Ao se avaliar a causa básica da morte fetal conforme a Classificação Internacional de Doenças e de Problemas Relacionados a Saúde, Décima Revisão - CID -10, mostram-se mais frequentes as causas relacionadas à P20 (Hipóxia intrauterina), somando 38\% (n=27), ao mesmo tempo em que 21\% ( $\mathrm{n}=15)$ das mortes apresentaram causa indeterminada (P95) (Tabela IV).

Tabela IV - Distribuição e frequência dos óbitos fetais segundo variáveis do natimorto. 10ª Região de Saúde do Ceará, Brasil, 2012 a 2013.

\begin{tabular}{|c|c|c|c|c|c|c|}
\hline \multirow{2}{*}{ Variáveis } & \multicolumn{2}{|c|}{2012} & \multicolumn{2}{|c|}{2013} & \multicolumn{2}{|c|}{ Total } \\
\hline & $\mathbf{n}$ & $\%$ & n & $\%$ & $\mathbf{n}$ & $\%$ \\
\hline \multicolumn{7}{|l|}{ Morte em relação ao parto } \\
\hline Antes & 26 & 83,8 & 33 & 82,5 & 59 & 83,1 \\
\hline Durante & 3 & 9,7 & 4 & 10,0 & 7 & 9,8 \\
\hline Sem informação & 2 & 6,5 & 3 & 7,5 & 5 & 7,1 \\
\hline \multicolumn{7}{|l|}{ Peso ao nascer } \\
\hline$<1.500$ & 5 & 16,1 & 16 & 40,0 & 21 & 29,5 \\
\hline $1.500-1.999$ & 5 & 16,1 & 11 & 27,5 & 16 & 11,3 \\
\hline $2000-2.499$ & 5 & 16,1 & 3 & 7,5 & 8 & 22,5 \\
\hline$>2.500$ & 14 & 45,2 & 8 & 20,0 & 22 & 31,0 \\
\hline Sem informação & 2 & 6,5 & 2 & 5,0 & 4 & 5,7 \\
\hline \multicolumn{7}{|l|}{ Causa Básica da Morte } \\
\hline P.20 (Hipóxia intrauterina) & 8 & 25,8 & 19 & 47,5 & 27 & 38,0 \\
\hline P.00 (Feto e recém-nascido afetados por afecções maternas) & 5 & 16,1 & 4 & 10,0 & 9 & 12,7 \\
\hline $\begin{array}{l}\text { P.02 (Feto e recém-nascido afetados por complicação da } \\
\text { placenta, do cordão umbilical e das membranas) }\end{array}$ & 6 & 19,3 & 6 & 15,0 & 12 & 17,0 \\
\hline P.95 (Morte fetal de causa não especificada) & 8 & 25,8 & 7 & 17,5 & 15 & 21,0 \\
\hline Outras causas & 4 & 13,0 & 4 & 10,00 & 8 & 11,3 \\
\hline
\end{tabular}

Fonte: Sistema de Informação sobre Mortalidade. Fichas de Investigação de Óbito Fetal

\section{DISCUSSÃO}

A taxa de mortalidade fetal na $10^{\mathrm{a}}$ Região de Saúde do Ceará mostrou-se alta no ano de 2013 (14,5/1.000 nascimentos), visto que, no mesmo período, a região Nordeste apresentou 13,3/1.000, sendo que, no estado do Ceará, a taxa esteve em 12 por mil nascimentos ${ }^{(5,6)}$.

As taxas de natimortalidade revelam importantes informações sobre as condições de saúde, assistência pré-natal e parto em uma determinada região ${ }^{(5,14)}$. No Brasil existem poucos estudos sobre o óbito fetal analisado isoladamente. Na literatura, é vinculada à mortalidade infantil como um todo, restringindo-se ao período perinatal (óbitos fetais acrescidos dos óbitos neonatais precoces $)^{(10)}$. Deste modo, reflete uma baixa visibilidade, interesse e compreensão de que esse evento, em grande parte, é evitável com a implantação e o acesso à serviços de qualidade de atenção materna ${ }^{(3,18)}$. A idade materna e a escolaridade estiveram associadas à natimortalidade, e assumiram especial relevância por sua inter-relação com outros fatores associados ao óbito fetal ${ }^{(11,13,19)}$.

No presente estudo, encontrou-se elevado percentual de óbitos fetais de mães com idade inferior a 30 anos $(52,1 \%$ dos casos). O resultado apresentado condiz com outras pesquisas, em que as mães com idade inferior a esta faixa etária apresentaram uma maior significância para natimortos ${ }^{(19,20)}$.

Em relação à escolaridade, no atual estudo, a maior frequência constituiu-se de mães com 8 a 11 anos de estudos (53,5\%), apresentando-se de forma divergente da literatura, uma vez que se aponta importante associação entre baixa instrução e óbito fetal $^{(11,12,19)}$.

Sobre as variáveis relacionadas à gestação, percebeu-se no presente estudo um maior número de óbitos fetais associados às gestantes primigestas. A nuliparidade e a existência de história reprodutiva anterior de morte perinatal são considerados fatores de risco gestacionais ${ }^{(21)}$, destacando-se a proporção de gestantes $(28,2 \%, n=20)$ com história gestacional de natimortos. Existe risco aumentado de recorrência de morte fetal em gestantes com histórico precedente de natimortalidade ${ }^{(12,22)}$.

Em referência ao início do pré-natal, cogita-se uma falha na captação precoce das gestantes, visto que na atual pesquisa, somente 49,3\% (n=35) iniciou o pré-natal antes do primeiro trimestre de gestação. A identificação precoce, até a $12^{a}$ semana de gestação, de todas as gestantes da comunidade para início do pré-natal, na Atenção Primária à Saúde, é um dos indicadores 
da qualidade do pré-natal na Atenção Básica. Ao mesmo tempo, promove a detecção e a intervenção precoce das situações de risco $^{(12,23)}$.

Pesquisas descrevem a associação entre doenças maternas e óbito fetal ${ }^{(11,12,19)}$. No presente estudo, quando avaliada a presença de complicações na gestação, sobressaiu-se a doença hipertensiva. As gestantes hipertensas apresentam risco maior para a ocorrência de morte fetal ${ }^{(12)}$, pois as alterações hipertensivas da gestação estão associadas às complicações graves fetais e maternas, além de um risco maior de mortalidade perinatal em virtude de alterações do fluxo placentário, determinadas pela doença ou tratamento ${ }^{(21)}$.

Os profissionais que prestam assistência às gestantes devem estar atentos à existência de fatores de risco e devem ter capacidade de avaliá-los dinamicamente, de maneira a determinar o momento em que a gestante necessitará de assistência especializada ou de interconsultas com outros profissionais. No momento em que se realiza uma intervenção, sobretudo no curso de uma gestação que possui maior chance de ter um resultado desfavorável, percebe-se uma ajuda na diminuição dos riscos a que estão expostos a gestante e o feto, e ainda, uma redução de possíveis consequências adversas ${ }^{(21,23)}$.

No que se refere às variáveis relacionadas ao parto, percebeu-se no estudo em questão que 33,8\% das gestantes buscaram o atendimento na hora do parto devido à ausência de movimento fetais. No entanto, não há informações suficientes que apontem o tempo entre a detecção e o atendimento no serviço de saúde. A ocorrência do óbito fetal antes do trabalho de parto se relaciona às condições maternas e à assistência prénatal ${ }^{(10,24)}$.

Os resultados apresentados no atual estudo mostram que a maioria das mortes ocorreu em gestações tardias ( $>28$ semanas de gestação), ou seja, com fetos com grande potencial de sobrevivência. Supondo que a qualidade da assistência pré-natal ainda não seja satisfatória, apesar de, no período de estudo, a cobertura populacional pelas equipes de Atenção Básica na $10^{\mathrm{a}}$ Região de Saúde do Ceará corresponder a 98,8\%. O elevado número de natimortos no final da gestação demostra a íntima relação entre esses óbitos e a qualidade dos serviços de saúde no atendimento às gestantes ${ }^{(10)}$.

Outra variável estudada na presente pesquisa foi o tipo de parto, indicando que a maioria $(67,6 \%)$ das concepções ocorreu por via vaginal. Esse dado é constatado pelo fato da via do parto recomendada em gestantes com óbito fetal ser a vaginal ${ }^{(25)}$. Porém, destaca-se o número de cesarianas (28,2\%), embora o óbito fetal não constitua indicação de cesárea, uma vez que, a princípio, a indução da via de parto deve ser a vaginal nos natimortos ${ }^{(25,26)}$. A cesariana no feto morto pode ser excepcionalmente indicada em algumas situações, como placenta prévia, cesáreas de repetição, descolamento da placenta, síndromes hipertensivas e outras doenças maternas associadas ${ }^{(21)}$. As taxas de cesárea têm aumentado em todo o mundo e particularmente no Brasil, pois essas indicações não são formais para realização do parto, mesmo com o concepto vivo. Assim, a cesariana em feto morto pode ser erroneamente justificada nessas situações, ressaltando que existe risco aumentado de feto morto subsequente ao parto por cesárea ${ }^{(21,25,26)}$.

Quanto às variáveis associadas ao natimorto, observou-se no atual estudo, maior frequência de óbitos fetais (83,1\%) ocorridos antes do trabalho de parto. Os resultados assemelham-se aos encontrados em países desenvolvidos, em que cerca de $10 \%$ das mortes fetais acontecem no momento do trabalho de parto ${ }^{(12)}$. Algumas classificações de evitabilidade, de acordo com a possibilidade de prevenção, têm sido utilizadas para análise dos óbitos infantis e fetais, sendo focadas as mortes causadas por problemas potencialmente tratáveis. Dentre elas, a Classificação de Wigglesworth, que propõe que uma proporção elevada de mortes anteparto refletem falha na assistência pré-natal ou condições maternas adversas ${ }^{(1,10,24)}$.

No que se refere ao peso ao nascer, verificou-se predominância de óbitos fetais com baixo peso na pesquisa em questão, resultado semelhante ao de outros estudos ${ }^{(11,13)}$. Destaca-se ainda que houve uma proporção significativa de natimortos (31\%) com peso igual ou acima de 2.500 gramas, um peso considerado com grande chance de sobrevivência, visto que, quanto maior a duração da gestação e/ou o peso, maior a viabilidade do feto, ou seja, maior o potencial de sobrevivência ${ }^{(8)}$ Portanto, uma vez que é fator isolado de maior importância para sobrevivência infantil ${ }^{(13)}$, toda análise de evitabilidade do óbito infantil e fetal devem ponderar o peso ao nascer.

Sobre as causas da morte, a hipóxia intrauterina (P.20 - CID 10) apresentou-se como a principal causa da morte fetal em $38 \%$ dos casos do atual estudo. Esse resultado é idêntico ao de uma pesquisa realizada em Salvador, Bahia, a qual revelou hipóxia em grande proporção entre os óbitos fetais ${ }^{(13)}$, correlacionando sua causa com falhas no manejo obstétrico, na assistência pré-natal e no parto, apontando qualidade inapropriada de atenção nos serviços de saúde ${ }^{(13)}$. No Brasil, as principais causas de óbito perinatal, dentre elas a asfixia perinatal, são as afecções passíveis de prevenção através de adequada atenção no prénatal e assistência ao parto ${ }^{(10)}$. No presente estudo, foram frequentes os casos em que o médico declarou somente "anóxia" ou "hipóxia", sem declarar a causa básica que desencadeou o agravo.

Outro fato importante a ser considerado em relação aos natimortos é o elevado percentual de morte fetal por causa mal definida (P.95 - CID 10), representando $21 \%$ dos óbitos encontrados no presente estudo. Logo, apontam-se sérias limitações no registro das causas e na qualificação da informação, comprometendo de forma significativa um melhor diagnóstico ${ }^{(8,9)}$. A ocorrência de morte fetal de causa não especificada deve ser avaliada para identificar sua etiologia e facilitar a adoção de medidas preventivas ${ }^{(24)}$.

O resultado encontrado na presente investigação também apontou a fragilidade no preenchimento da Declaração de Óbito e das Fichas de Investigação do Óbito Fetal, tendo em vista o elevado número de variáveis sem informação. É um achado 
inquietante, pois traz a reflexão do insuficiente conhecimento ou valorização da ação do preenchimento dos dados da DO e das fichas de investigação, já que os dados corretamente preenchidos poderiam ser válidos para identificar e analisar a magnitude da mortalidade, bem como, avaliar ações de saúde implementadas ${ }^{(1,27)}$.

A constituição de comitês de prevenção de óbitos institui a melhoria da apresentação dos dados e a análise das circunstâncias do óbito. Contudo, além disso, qualificar a informação é uma importante etapa para avaliação adequada do perfil da mortalidade por causas e fatores ${ }^{(28)}$.

Por fim, acredita-se que a realização de estudos sobre natimortalidade somam-se significativamente para contribuir no planejamento das ações de assistência à saúde materna e de evitabilidade das mortes fetais.

\section{CONCLUSÃO}

A situação de saúde quanto à mortalidade fetal evidenciou aumento no período analisado, alta taxa de óbitos no ano de 2013 (14,5/1.000 nascimentos), cujas mães eram jovens, primigestas e com complicações gestacionais, com mortes ocorridas antes do parto, baixo peso e hipóxia intrauterina.

Embora o óbito fetal ocasione perda que implica angústia na vida da mãe, sua ocorrência ainda é associada a uma eventualidade que não pode ser prevenida e tem sido negligenciada pelos serviços de saúde.

\section{REFERÊNCIAS}

1. Ministério da Saúde (BR). Manual de vigilância do óbito infantil e fetal e do comitê de prevenção do óbito infantil e fetal. $2^{\mathrm{a}}$ ed. Brasília: Ministério da Saúde; 2009.

2. Brasil. Portaria $\mathrm{n}^{\mathrm{o}} 72$, de 11 de janeiro de 2010. Estabelece que a vigilância do óbito infantil e fetal é obrigatória nos serviços de saúde (públicos e privados) que integram o Sistema único de Saúde. Brasília: Ministério da Saúde; 2015 [acesso em 2015 Abr 20]. Disponível em: http://bvsms.saude.gov.br/bvs/saudelegis/gm/2010/prt0072_11_01_2010.html

3. Bernis L, Kinney MV, Stones W, Hoope-Bender TP, Vivio D, Leisher SH, et al. Stillbirths: ending preventable deaths by 2030. Lancet. 2016;387(10019):703-16.

4. Ministério da Saúde (BR), Departamento de Informática do SUS (DATASUS). Estatísticas vitais. Monitoramento de eventos prioritários de mortalidade (SVS/DANTPS): Painel de monitoramento da mortalidade infantil e fetal [acesso em 2017 Fev 15]. Disponível em: http://svs.aids.gov.br/dashboard/mortalidade/infantil.show.mtw

5. Ministério da Saúde (BR), Departamento de Informática do SUS - DATASUS. Estatísticas vitais. Mortalidade: óbitos fetais [acesso em 2015 Maio 10]. Disponível em: http://www2.datasus.gov.br/DATASUS/index.php?area=0205\&VObj=http:// tabnet.datasus.gov.br/cgi/deftohtm.exe?sim/cnv/pfet10

6. Secretaria de Saúde do Estado do Ceará. Sistema de Informação. Tabnet. Mortalidade. Fortaleza [acesso em 2015 Maio 10]. Disponível em: http://extranet.saude.ce.gov.br/scripts/deftohtm.exe?sim/obito.def

7. Frias PG, Navarro LM. Crianças: sujeitos de direito e sua vulnerabilidade. In: Bittencourt DAS, Dias MAB, Wakimoto MD. Vigilância do óbito materno, infantil e fetal e atuação em comitês de mortalidade. Rio de Janeiro: Fiocruz; 2013. p. 91-133.

8. Lawn JE, Blencowe H, Pattinson R, Cousens S, Kumar R, Ibiebele I, et al. Stillbirths: Where? When? Why?: how to make the data count? Lancet. 2011;377(9775):1448-63.

9. Bhutta ZA, Yakoob MY, Lawn JE, RIZVI A, Friberg IK, Weissman E, et al. Stillbirths: what difference can we make and at what cost? Lancet. 2011; 377(9776):1523-38

10. Lansky S. Mortalidade Fetal: mortes invisíveis e evitáveis. In: Bittencourt DAS, Dias MAB, Wakimoto MD. Vigilância do óbito materno, infantil e fetal e atuação em comitês de mortalidade. Rio de Janeiro: Fiocruz; 2013. p. 123-33.

11. Andrade LG, Amorim MM, Cunha AS, Leite SR, Vital SA. Fatores associados à natimortalidade em uma maternidade escola em Pernambuco: estudo caso-controle. Rev Bras Ginecol Obstet. 2009;31(6):285-92.

12. Klein CJ, Madi JM, Araújo BF, Zatti H, Bosco DSDB, Henke CN, et al. Fatores de risco relacionados à mortalidade fetal. Rev AMRIGS. 2012;56(1):11-6.

13. Jacinto E, Aquino EML, Mota ELA. Mortalidade perinatal no município de salvador, Bahia: evolução de 2000 a 2009. Rev Saúde Pública 2013;47(5):846-53. 
14. Lansky S, França E, Leal MC. Mortalidade perinatal e evitabilidade: revisão de literatura. Rev Saúde Pública. 2002;36(6):759-72.

15. Secretaria de Saúde do Estado do Ceará. Revisão do Plano Diretor de Regionalização das Ações e Serviços de Saúde - PDR do Estado do Ceará 2014 [acesso em 2015 Maio 15]. Disponível em: http://www.saude.ce.gov.br/index.php/downloads/ category/99-plano-diretor-de-regionalizacao-pdr-2014

16. Instituto Brasileiro de Geografia e Estatística - IBGE. Informações sobre municípios do Ceará [Internet]. Rio de Janeiro: IBGE; 2015 [acesso em 2015 Fev 10]. Disponível em: http://www.cidades.ibge.gov.br/xtras/uf.php?lang=\&coduf=23\&se $\operatorname{arch}=$ ceara

17. Ministério da Saúde (BR), Secretaria de Gestão Estratégica e Participativa. Decreto no 7.508, de 28 de junho de 2011 : Regulamentação da Lei no 8.080/90. Brasília: Ministério da Saúde; 2011.

18. Bhutta ZA, Das JK, Bahl R, Lawn J E, Salam RA, Paul VK, et al. Can available interventions end preventable deaths in mothers, newborn babies, and stillbirths, and at what cost?. Lancet. 2014;384(9940):347-70.

19. Vieira MM, Camargo SE, Ceglio WQGW, Almeira MH, Batista TS, Freitas PF. Dificuldades para a identificação da causa do óbito fetal: como resolver? Rev Bras Ginecol Obstet. 2012;34(9):403-8.

20. Chiavegatto FADP, Laurenti R. O sexo masculino vulnerável: razão de masculinidade entre os óbitos fetais brasileiros. Cad Saúde Pública. 2012;28(4):720-8.

21. Ministério da Saúde (BR). Gestação de alto risco: manual técnico. 5a ed. Brasília: Ministério da Saúde; 2012.

22. Getahun D, Lawrence JM, Fassett MJ, Strickland G, Koebnick C, Chen W, et al. The association between stillbirth in the first pregnancy and subsequent adverse perinatal outcomes. Am J Obstet Gynecol. 2009;201(4):378.e1-6.

23. Ministério da Saúde (BR). Atenção ao pré-natal de baixo. Brasília: Ministério da Saúde; 2013.

24. Assis HM, Siviero PCL, Drumond EF, Machado CJ. Óbitos fetais sob o prisma da evitabilidade: análise preliminar de um estudo para o município de Belo Horizonte. Cad Saúde Colet (Rio J). 2014;22(3):314-7.

25. Sampaio GA, Souza ASR. Indicação de cesarianas em óbito fetal. Rev Bras Ginecol Obstet. 2010;32(4):169-75.

26. Nascimento MI, Cunha AA, Oliveira SRSM. Manejo Clínico na indução de parto de feto morto: avaliação da incidência e condições associadas à cesariana. Rev Bras Epidemiol. 2014;17(1):203-16.

27. Cascão AM, Rocha PMM, Wakimoto MD, Bittencourt SDA. Instrumentos para registro de óbitos e nascimentos e seus sistemas de informação em saúde. In: Bittencourt DAS, Dias MAB, Wakimoto MD. Vigilância do óbito materno, infantil e fetal e atuação em comitês de mortalidade. Rio de Janeiro: Fiocruz; 2013. p. 137-69.

28. Santos SPC, Lansky S, Ishitani LH, França EB. Óbitos infantis evitáveis em Belo Horizonte: análise de concordância da causa básica, 2010-2011. Rev Bras Saúde Mater Infant. 2015;15(4):389-99.

\section{Endereço para correspondência:}

Katherine Jeronimo Lima

Universidade Estadual do Ceará - UECE

Programa de Pós-Graduação em Saúde Coletiva - PPSAC

Avenida Dr. Silas Munguba, 1700

Bairro: Itapery

CEP: 60.740-000 - Fortaleza (CE) - Brasil

E-mail: katherine.jeronimo@gmail.com 